\title{
La inteligencia colectiva y la responsabilidad social y política del investigador. "Del yo al nosotros y del nosotros al todo"
}

Luis Eduardo Wilches Mahecha*

Ricardo Javier Jiménez Silva*

Recibido: 26 de septiembre de 2013 - Aprobado: 30 de enero de 2014

\section{Resumen}

El presente artículo busca avivar el diálogo político, ético, ecológico y participante, frente a la permanente necesidad de deconstruir y reconstruir propuestas, tras la búsqueda de epistemes que favorezcan la generación de conocimientos y comprensiones, relacionadas con un enfoque transdisciplinar denominado inteligencia colectiva. Esto a partir de metodologías, métodos e instrumentos propios, que aporten elementos para la construcción de una apuesta diferente ante el modelo de desarrollo imperante, y a partir de la práctica colectiva para la búsqueda de soluciones concretas que distan de posiciones y debates totalitaristas, de manera que se propicien escenarios de encuentro con diálogos y saberes innovadores para superar, concertada y estratégicamente, problemas contemporáneos de nuestra sociedad.

Palabras clave: inteligencia colectiva, otredad, redes, gestión del conocimiento, multiculturalidad.

Sociólogo y Magíster en estudios culturales de la Universidad Nacional de Colombia, se desempeña como docente de la Universidad Nacional Abierta y a Distancia -UNAD. Dirección postal: Calle 14 Sur No. 14-23, Bogotá, Colombia. Correo electrónico: luise.wilches@unad.edu.co.

** Magister en Educación de la Universidad Pedagógica de Colombia, docente de la Universidad Nacional Abierta y a Distancia - UNAD. Dirección postal: Calle 14 Sur No. 14-23, Bogotá, Colombia. Correo electrónico: ricardo.jimenez@ unad.edu.co. 


\title{
Collective intelligence and social and political responsibility of the researcher. "From I to us and from I to the we to all"
}

\author{
Luis Eduardo Wilches Mahecha \\ Ricardo Xavier Jiménez Silva
}

\section{Abstract}

The present article pretends to sharpen the political, ethical, ecological and participant dialogue, to address the permanent need of constructing and rebuild proposals, in the search of epistemes that favor the generation of knowledge and understandings related with an trans-disciplinary approach called collective intelligence from methodologies methods and tools, that provide elements for the construction of different proposal in regards to the prevailing model of development from collective practice to find specific solutions different from totalitarian positions and proceedings in ways that support encounter scenarios with dialogues and innovative knowledge to overcome, concertedly and strategically, contemporary problems of our society.

Keywords: Collective Intelligence, Otherness, Networking, Knowledge Management, Multiculturalism.

Sociologist and Master in Cultural Studies from the Universidad Nacional de Colombia, is a teacher at the Universidad Nacional Abierta y a Distancia -UNAD. Address: Calle 14 Sur No. 14-23, Bogotá, Colombia. Email: luise.wilches@unad. edu.co.

** Master in Education from te Universidad Pedagógica de Colombia, is a teacher at the Universidad Nacional Abierta y a Distancia -UNAD. Address: Calle 14 Sur No. 14-23, Bogotá, Colombia. Email: ricardo.jimenez@unad.edu.co. 


\title{
L'intelligence collective et la responsabilité sociale et politique du rechercheur. «De moi au nous et de nous au tout»
}

\author{
Luis Eduardo Wilches Mahecha \\ Ricardo Javier Jiménez Silva
}

\section{Résumé}

Le présent article cherche à aviver le dialogue politique, éthique, écologique et participant, en face à la nécessité permanente de de-construire et reconstruire des propositions, à partir de la recherche d'épistèmes que favorisent la génération de connaissances et de compréhension. Elles se relient avec un point de vue transdisciplinaire dénommé l'intelligence collective, à partir des méthodologies, des méthodes et des instruments propres qui apportent des éléments pour la construction d'un défi, en face du modèle de développement régnant. À partir de la pratique collective on recherche de solutions concrètes qui sont éloignées des positions et des débats totalitaristes. De cette manière, elles favorisent des scenarios de rencontre avec des dialogues et des savoirs innovateurs pour surpasser, concertée et stratégiquement, des problèmes contemporains de notre société.

Mots Clés: Intelligence Collective, Otredad, des Réseaux, la Gestion de la Connaissance, Multiculturalidad.

5 Sociologue et Magister en études culturelles de l'Universdad Nacional de Colombia, est un professeur à l'Universidad Nacional Abierta y a Distancia -UNAD. Adresse: Calle 14 No. 14-23 Sur, Bogotá, Colombia. Email: luise.wilches@unad. edu.co.

6 Magister en éducation de l'Universidad Pedagógica de Colombia, est un professeur à l'Universidad Nacional Abierta y a Distancia -UNAD. Adresse: Calle 14 No.14-23 Sur, Bogotá, Colombia. Email: ricardo.jimenez@unad.edu.co. 


\section{Introducción}

Hay un inminente fracaso de los postulados que pretendían desarrollar los paradigmas de la modernidad y la posmodernidad, con sus propuestas de Estado-nación, civilización y progreso, sumadas a la estrategia economicista del neocapitalismo con su idea del libre mercado -que fueron planteados como pretextos para dar solución a condiciones de exclusión social, inequidad, deterioro ambiental, entre otros, y que contrariamente sus resultados han puesto en riesgo la multidiversidad social, cultural y política, y cuánto más la supervivencia humana-.

Ante lo anterior, irrumpe como necesidad la reflexión y la acción hacia un nuevo proyecto de humanidad -cuya emergencia requiere de creatividad y recursividad- que sea propio de acciones políticas concretas, desde la transformación del sujeto social hacia un sujeto que piense su relación con lo colectivo, y en el que se infiera la incorporación sobre su relación con los entornos naturales, y se presenten sus búsquedas más profundas sobre el sentido de la vida y su estar en un mundo, con multiplicidad de facetas y tipos de vida.

Se espera que esta reflexión se promueva de forma afectiva, pacífica y sostenida, para alcanzar, ante el paradigma neoliberal, procesos transicionales hacia un nuevo desarrollo social, pues la globalización de este paradigma se ha convertido en una postura hegemónica que raya en el fundamentalismo, para avanzar hacia una nueva mirada sobre el desarrollo humano, cruzado por concepciones incluyentes que repiensen el tema de lo glocal, centradas en el respeto por la vida y la naturaleza como escenario donde esta se dimensiona.

Pensar estas aristas requiere la revisión de planteamientos efectuados por pensadores de toda índole, como faros que encaminen reflexiones movilizadoras de lo íntimo, pues el sujeto no se halla en lo colectivo hasta que encuentra su relación consigo mismo y reconoce éticamente la necesidad de transitar hacia el beneficio colectivo, sin abandonar sus particularidades, que lo hacen único y por ende aportante como cimiento para un todo social.

Ejemplos de esto se encuentran en expresiones sociales que registran acopio de las realidades que envuelven las dinámicas de los cuerpos sociales, en las que las comunidades son interpretadas y reflejadas en manifestaciones estéticas. Consideramos que un claro ejemplo con estas características es la introducción a la interpretación de una canción, en la que con un sentir ecológico, político y ético, se denuncia el deterioro ambiental causado por un tipo de consciencia humana: “...es una canción que con mucha amargura y un tanto de vergüenza yo quiero dedicar a esos canallas que queman nuestros bosques, ensucian nuestras aguas, envenenan nuestros alimentos y se enriquecen con las miserias ajenas"1.

1 Introducción realizada por Joan Manuel Serrat en su concierto en Alicante (España) en 1983, antecediendo su interpretación del tema Pare. 
Otro ejemplo -surgido en una latitud diferente-, que propone ampliar posibilidades de conciencia con sus aforismos, se evidencia en la sabiduría taoísta cuando plantea que el aleteo de las alas de una mariposa puede provocar un Tsunami al otro lado del mundo. Sencillamente, se muestra la relación de una acción, cualquiera que sea, como desencadenante de movimientos en la naturaleza, y que al llevarlos al plano social, sus posibilidades trasformadoras pasan inadvertidas.

Una inquietud que surge tiene que ver con los factores que pueden estar obstaculizando que pensamientos diversos y hasta divergentes incidan en otras latitudes, dada la imposición que presentan ciertas dinámicas de construcción del desconocimiento. Estas acciones parecerían estar concebidas de manera que direccionan a las personas a mantenerse en un estado casi catatónico, ante la influencia de gran parte de los medios de comunicación y el uso que les han imprimido las estructuras de poder, que pese a su visibilidad, para la mayoría de la sociedad parecen invisibles, pues con la construcción de discursos que movilizan lo que pretenden que sea el sentido de la vida, las distancian ante lo que el grueso de estas personas esperaría que fueran.

Sobre esto, Noam Chomsky (s. f.) ya había efectuado un llamado, mostrando que:

...la literatura sobre la industria de la comunicación pública nos proporciona una rica e instructiva retahíla de instrucciones sobre cómo implementar el 'nuevo espíritu de la época' mediante la creación de necesidades, o bien a través de 'regir la opinión pública del mismo modo que un ejército rige los cuerpos de sus soldados', e induciendo a una 'filosofía de la futilidad' y a una carencia de objetivos en la vida, concentrando la atención humana en 'las cosas más superficiales, las referidas en gran parte al consumo de moda'. Si esto es posible, entonces la gente aceptará su insignificante y subordinada vida, apropiada para ellos, y así se dejarán de ideas subversivas, de tomar el control de sus vidas.

Pero no son nuevas estas reflexiones sobre la influencia del poder y sus verdades, que se encuentran presentes a lo largo de la historia de la humanidad, y no pretenden ser la idea central de este apartado. Antes bien, la cuestión es si estamos ante la búsqueda de una sociedad que quiera o no regir sus destinos de forma libre, pero coherente con la vida como centro y fin último de su movilización; o si acaso estamos ante una condición humana que prefiera seguir bajo un totalitarismo ideológico, casi impuesto por la apatía de pensar diferente, y peor aún, de negarnos la posibilidad de pensar colectivamente, en lo posible, para comprender que no estamos solos ante estas búsquedas.

Pensamos en la vida, y en la muerte como opuesta a ella, para observar si como especie humana que pretende luchar para evitar su extinción, podemos preguntarnos en dónde quedó esa inteligencia que la evolución nos llevó a incorporar a modo de instinto de supervivencia, y que frente a los aconteceres de la degradación y depredación entre nosotros mismos y la naturaleza de la cual formamos parte, pareciera que se ha reducido también como manifestación 
colectiva, ante cierto tipo de ceguera de pensamiento, no por ausencia de ideas, sino por inexistencia de puentes que permitan conectarnos con otros, que tal vez sin saberlo tienen concepciones y proyecciones de vida afines sobre estas cuestiones.

Un ejemplo que pareciera opuesto a lo que la academia considera válido es lo que encontramos en una película infantil animada, que muestra cómo un banco de peces, luego de ser atrapado por una red, había llegado al punto de aceptar su destino, hasta que aparece un pez con características únicas que lo hicieron marcar la diferencia: “tenía una búsqueda" que motivó al banco de peces resignados a asumir un movimiento conjunto que movilizara una fuerza en contrasentido a la red, para que se dieran cuenta de que, como especie, estaban dispersos en el sentido de supervivencia, y así se liberarían de lo que momentos antes se consideraba inevitable... su muerte.

Un elemento que necesita ser leído con mayor detenimiento es el que subyace a lo que Edgar Morín (2007) plantea:

Por muy desarrollado que esté en el animal el sector de inventiva individual, la inteligencia específica continúa siendo el determinante esencial: la afirmación de sí que contradice con su aparición la jerarquía de la especie y su 'unicidad', solo surge con la conciencia de sí.

Esta conciencia de sí es un aspecto que exige reconocer que es posible que surja la inteligencia hacia lo colectivo en la medida en que se desarrolle, simultáneamente, una conciencia propia en el individuo. Así como una conciencia de los aportes que ambas podrían entregar al colectivo, pues sin ellas no existirían nodos que permitieran encontrar puntos de referencia para la articulación, de manera que se presenta un tipo de sinapsis social, como un símil de la interacción que las neuronas presentan en el sistema que conocemos como cerebro.

La pregunta es cómo es posible que logren mantener a tal cantidad de personas bajo una perspectiva de desconocimiento sobre alternativas para una vida digna y con calidad, en las que lo económico solo sea un elemento facilitador y no totalizador en la construcción de sentido y de subjetividad de los seres humanos y de las conciencias colectivas.

¿Acaso las conciencias integrativas de esos "otros" saberes no sintetizan de manera profunda la gran crisis social, política, ética y ecológica de nuestra contemporaneidad? Y tras una pregunta surgen otras: acaso la emergencia de nuevas identidades, sabidurías y nuevas subjetividades, y de los movimientos sociales, producidos por la transicionalidad permanente y connatural de nuestra sociedad, o las sabidurías ancestrales originarias producto de miles de años de experiencia, vivencia y cualificación, ¿tienen algo que aportar y dialogar con la producción científica y académica?

Esto nos lleva a una nueva pregunta en términos políticos y éticos: ¿es la universidad, y sus instancias de investigación en particular, el único escenario 
legitimador de "la verdad" y el único lugar sagrado de solución de los problemas contemporáneos? Como correlación, este interrogante permite retomar dos de las preguntas planteadas por Foucault en el artículo "Subjetividad y verdad" (1981): “¿Cómo la experiencia que se puede hacer de sí mismo y el saber que de ello se forma han sido organizados a través de ciertos esquemas?, ¿cómo se han definido, valorado, aconsejado o impuesto estos esquemas?"

Pero en una arquitectura piramidal ${ }^{2}$ como la nuestra, claramente excluyente y perfilada para que cada elemento o parte funcional y vital de esa estructura potencie el estado de cosas o la naturalización de la realidad o la verdad, ¿será que la universidad, siendo solo un escenario más en el que se genera conocimiento de la sociedad, podría priorizar su sentido social, humano y ecológico por sobre los estándares internacionales de competencia y jerarquización, diseñados y concebidos para el fortalecimiento de esa estructura piramidal?

¿No se han manifestado, por ejemplo, en América Latina con la investigaciónacción participativa (IAP), o en Estados Unidos con la etnometodología, o en India con los estudios subalternos, en los que las ciencias humanas y sociales logran dimensionar más equitativamente las sabidurías de las comunidades, entendiéndolas y aceptándolas como legítimas y con capacidad de dialogar con los conocimientos científicos para la búsqueda de soluciones a los problemas locales de esas comunidades?

Y estas experiencias, ¿no han comenzado a visibilizar, y sobre todo, a validar y a legitimar ante las comunidades, que existen sabidurías populares, no académicas, que están en capacidad de dialogar paralelamente con el conocimiento científico, y que tienen conocimientos, informaciones y metodologías para aportar a la solución de dichos problemas?

Y en contexto meramente científico, acaso el fenómeno cada día más decidido de acercamiento entre las disciplinas para poder comprender las nuevas y complejas realidades contemporáneas, y entre las ciencias duras y las blandas, ¿no es un síntoma de que la percepción de la vida en nuestra era está cambiando hacia la integralidad y las comprensiones eco-sistémicas y bio-éticas? ¿No es muy sintomático que la ciencia se haga cada vez más consciente de esta nueva percepción integrativa -como por ejemplo, la física o la biología, hoy por hoy las disciplinas líderes en abrir la puerta a una ampliación de consciencia integrativa-, que se aproxima cada vez más a una mirada espiritual o integrativa frente a los problemas ambientales, eco-sistémicos y bio-éticos, entre el hombre ("el otro") y la naturaleza ("lo otro") que desde hace miles de años, anteriores a la ciencia moderna y a la academia, ya habían desarrollado las comunidades en múltiples latitudes alrededor del mundo? ¿No existen ahora tendencias de análisis-síntesis en casi todos los intentos de generación de conocimiento?

2 En contraposición a la representación de una pirámide para mostrar la estructura de la sociedad contemporánea heredada de la cultura occidental, Jean François Noubel propone la idea de una estructura holomidal (que surge de la unión de las palabras holístico y piramidal), en la que no existe una élite y una gran base definidas, sino que se representa con la idea de un rizoma. 
Acercarnos a la comprensión profunda del enfoque transdisciplinar denominado inteligencia colectiva, propuesto por el francés Jean François Noubel y que llamó la revolución invisible, es -más que una moda académica, que genera puntajes importantes en los rankings internacionales de las universidades o en el peldaño para el incremento de las ganancias financieras de los autores o las instituciones que la promueven- la concreción de una propuesta práctica y aplicada para generar procesos de cambio hacia esa nueva consciencia integrativa frente a la vida.

La propuesta de la inteligencia colectiva es una filosofía de vida y de cambio que tiene en cuenta el contexto transicional que de constituir un sujeto social -ese ser competitivo, depredador y aniquilador de lo diferente $u$ otro- se traslada a la constitución de un ser-humano-espiritual, que se fundamenta en una ética integrativa. En resumen, la transición y el desarrollo consciente y espontáneo a la vez de un nuevo "humano" que se vincula integrativamente consigo mismo, con "los otros", con "lo otro" y con el universo de "lo trascendental o espiritual", como la narración de Sócrates en su apología ante los tribunales de Atenas: "Os ocupáis de vuestras riquezas, de vuestra reputación y de los honores; pero no os preocupáis ni de vuestra virtud, ni de vuestra alma".

En este orden de ideas, ¿cuál es el compromiso del investigador y del educador en un ejercicio de cambio como el que se presenta? ¿Será la transformación de los sujetos sociales en seres-humanos-espirituales lo que provocaría una transformación de la institucionalidad educativa, a partir de generar nuevas políticas públicas y colectivas? $\mathrm{O}$ ¿será que dependemos de las instancias institucionalizadas legitimadoras de verdades, con base en los desarrollos de investigación y estudios especializados patrocinados por los gobiernos locales o globales, que procesarán esa transformación?

Se hace necesario presentar el estado de situación en el que nuestros grupos de trabajo se encuentran en su proceso de investigación sobre lo que queremos denominar inteligencias integrativas, en plural, y proponer este concepto desde el principio central de nuestro trabajo que es del yo al nosotros y del nosotros al todo.

\section{1 \\ Del yo al nosotros y del nosotros al todo": acuerdos mínimos para dialogar sobre las inteligencias integrativas}

A manera de aclaración del título de este apartado, creemos necesario comentar que en el ejercicio dialógico que venimos desarrollando en la Universidad Nacional Abierta y a Distancia (UNAD), ${ }^{3}$ se está tratando de posicionar el campo

3 Particularmente en la Escuela de Ciencias Sociales, Artes y Humanidades (ECSAH), con tres grupos de trabajo: el Observatorio de la Participación Social (OPS); el equipo de diseño y puesta en marcha del Programa de Sociología, y el Grupo de Investigación Experimental en Inteligencias Colectivas (GIEIC), equipo con el que estamos tratando de posicionar el campo de inteligencias integrativas. 
de inteligencias integrativas, en el cual se incorporarían los enfoques de la inter-creatividad ${ }^{4}$, las multitudes inteligentes ${ }^{5}$, la sabiduría de las multitudes ${ }^{6}$, la web 2.0 inteligente o la inteligencia conectiva ${ }^{7}$, la inteligencia colaborativa ${ }^{8}$, la co-inteligencia, la inteligencia comunitaria (en proceso de construcción) y la inteligencia colectiva, que es la que, en nuestra opinión, tiene un mayor potencial de desarrollo científico y aplicativo.

Antes de revisar muy someramente cada uno de los enfoques propuestos antes, es muy importante resaltar que existen dos vertientes generalizadas en estas propuestas integrativas: la primera es la que emerge de la interacción virtual, mediada por internet y las nuevas TIC, y que se presenta más próxima a la necesidad de generar comunidades virtualizadas mediatizadas, es decir, que el fin central es el desarrollo tecnológico y el diseño de instrumentos medios para el logro de estos fines. La segunda vertiente es la que propone al internet y a las TIC como una estrategia más dentro de la necesidad de intercomunicar a una inmensa emergencia de actores sociales que, mediados por sus nuevas necesidades sociales sentidas, implementan desarrollo de mediación y no de medios como tal.

\section{Antecedentes de la propuesta científica de la inteligencia colectiva}

Desde finales de los noventa, el científico social Pierre Lévy ${ }^{9}$ desarrolló el contexto epistémico y axiológico de una "disciplina” que proponía la necesidad de abrir el conocimiento científico a un diálogo con las emergentes comunidades virtuales de las nuevas tecnologías y la web. En su texto Inteligencia colectiva: una antropología del ciberespacio (2004), Lévy acuñó la esencia de esta propuesta integrativa de comprensión de los nuevos fenómenos sociales y humanos. En el 2004, el maestro francés Jean François Noubel ${ }^{10}$ escribió el texto alternativo-

4 Tomado del blog "Lo Imaginado". Recuperado el 16 de mayo de 2007, de http://loimaginado.blogspot.com/2007/05/ el-concepto-de-intercreatividad-y-el.html

5 El desarrollo en esta línea está cimentado en la construcción conceptual de Howard Rheingold, quien en su obra Multitudes inteligentes. La próxima revolución social (Smart Mobs) (2004), postula que las comunidades virtuales se generan al estilo de un ecosistema de subculturas y grupos que, en su génesis y a pesar de no poseer un vínculo per se, se gesta de manera espontánea y cuando las tecnologías de la comunicación amplían los talentos humanos de cooperación.

6 James Surowiecki, columnista de la revista The New Yorker y autor del libro Cien mejor que uno (título en español), añade nuevos aportes al concepto de intercambio y generación de conocimientos individuales a una esfera colectiva.

7 Derrick de Kerckhove (1998) define la inteligencia conectiva como "una condición de la mente que nace de la asociación espontánea o deliberada de numerosas personas en grupos".

8 La inteligencia colaborativa puede clasificarse de acuerdo con el grado y el tipo de colaboración que los individuos produzcan sobre el producto final.

9 El profesor francés de ascendencia judía Pierre Lévy tiene maestría en historia de la ciencia y doctorado en sociología y ciencias de la información de la Universidad de la Sorbona. Desde el 2002, es titular de la cátedra de Investigación en Inteligencia Colectiva de la Universidad de Ottawa (Canadá). Miembro de la Royal Society de Canadá.

10 El doctor francés Jean François Noubel es codirector y fundador del Collective Intelligence Research Institute (CIRI), con sede principal en Francia. Es cocreador de la Red Internacional de Inteligencia Colectiva The Transitioner. Su texto "El 
científico Inteligencia colectiva: la revolución invisible, en el que la presenta como una disciplina que posibilita acciones concretas para la búsqueda de soluciones alternativas en el nuevo marco de cambio profundo de la sociedad.

Un ejemplo concreto de compromiso efectivo con su planteamiento "en acción" es el desarrollo de la Red Internacional de Inteligencia Colectiva The Transitioner y la presentación del texto "El futuro del dinero", en el que plantea la viabilidad de una nueva idea de economía holomidal (en contraposición de piramidal propone la idea de una nueva estructura holística-holomidal), en la que resalta la necesidad de comprender las nuevas inteligencias, conciencias y sabidurías colectivas.

La inteligencia colectiva es presentada por Lévy y Noubel, entre otros autores, como el desarrollo de una nueva disciplina alternativa fundamentada en el concepto de la holomidalidad, es decir, una estructura opuesta a la piramidal, y en donde la esencia de las prácticas sociales, entre ellas la académica, debe estar basada en una mirada profundamente holístico-integrativa, que supera con ello las arquitecturas invisibles ${ }^{11}$ de la dominación del sistema hegemónico piramidal. Desde el uso respetuoso de la palabra y el amor por sí mismo reflejado en prácticas de relajación, meditación y autocontrol de las emociones, pasando por elementos claves de la programación neurolingüística (PNL), hasta la aplicación de las teorías de la física cuántica y la biología molecular, en estrecha relación con el universo académico de las ciencias sociales y las humanidades.

Desde esta perspectiva, la inteligencia colectiva se convierte en un potente instrumento de generación de pensamientos y sabidurías integrativas propias, y posiciona una actitud profunda de de-colonización autocrítica del pensamiento, que propicia el diálogo entre saberes interdisciplinares y a-disciplinares (sabidurías ancestrales y milenarias, campesinas y alternativas, etc.), con lo que se posiciona como un valioso instrumento de transversalidad sistémica en donde sea implementada. Así mismo, permite construir en conjunto una forma de ser sujetos constructores de nuevas realidades, con la capacidad de transformar los diversos ámbitos del ser en sus diversas dimensiones complejas.

La inteligencia colectiva, entonces, propende a un ejercicio juicioso por cualificar nuestra conciencia como especie humana, y propende a un posicionamiento que, frente a la gran transición contemporánea, genera mecanismos efectivos para posibilitar el paso del enfoque epistemológico del constructivismo (ecosocial) de la academia tradicional a un paradigma integrativo (holomidal), y el componente educativo, particularmente el virtual, se constituye justamente en el eje central de ese proceso de transición. 


\section{Los "otros": ¿ ¿estamos condenados a la inteligencia individual o la incapacidad colectiva?}

El nuevo campo transdisciplinar denominado inteligencia colectiva se basa en los desarrollos de las nuevas tecnologías y en los cambios de perspectiva del relacionamiento entre el vínculo social y el conocimiento, que vaticinan la necesidad de comprender al "otro" ya no como nuestro enemigo o con el que hay que competir, sino como una fuente de conocimiento, vivencia y cooperación mutua. Su base y meta es "el reconocimiento mutuo y el enriquecimiento de individuos, en vez del culto de comunidades fetichistas e hiperestetizadas" (Lévy, 2004). La nueva tendencia es, más bien, la creación de una inteligencia universalmente distribuida, en la que nadie sabe todo y todos saben algo.

El lineamiento de la inteligencia colectiva, entendida como una revolución invisible, fue propuesto por Jean François Noubel, director del Collective Intelligence Research Institute (CIRI) ${ }^{12}$, y se presenta como una clara estrategia para el re-encuentro con "los otros", pero sobre todo para la "legitimación" del otro. Es el instante en que el otro y yo no somos dos, sino uno, en un propósito. El CIRI, organización internacional independiente con sede en Francia, viene llevando procesos de investigación y desarrollo práctico de inteligencia, sabiduría y conciencia colectiva, en los que todos los materiales y resultados producidos son directamente ofrecidos al “dominio" público.

En las inteligencias integrativas, el objetivo central es encontrar formas prácticas de "legitimar" al otro y a lo otro, para posibilitar de nuevo el "re-encuentro" humano y el de los individuos con el medio ambiente. Esta idea-fuerza, en el contexto de la dimensión de la educación, es la base para luchar contra la fragmentación generada en Occidente entre el conocimiento científico y las "otras" sabidurías.

El enfoque transdisciplinar que propone la inteligencia colectiva aplicada a la potenciación de procesos de integratividad, cohesión y articulación, se convierte en un instrumento concreto que posibilita ampliar la consciencia de la institución universitaria y, en particular, de los investigadores como generadores de pensamientos y sabidurías propios para fomentar actitudes, intenciones y escenarios adecuados y paralelos de diálogo entre la academia y las sabidurías otras, con el fin de identificar, conocer y contribuir con la solución de las nuevas necesidades sociales sentidas. Todo esto con la comprensión participativa de las sabidurías comunitarias, ancestrales y alternativas, en el reconocimiento de la emergencia permanente de las nuevas identidades, del accionar colectivo, de las nuevas formas de asociatividad, y de ampliar la comprensión de las nuevas subjetividades que, en este estado transicional a un nuevo paradigma, constituyen el núcleo central de esta revolución invisible. 
Podríamos sintetizar la comprensión de este campo -la síntesis es uno de los objetivos centrales de la construcción de sabidurías para el pensamiento integrativo- en cuatro de las más revolucionarias y profundas semillas para la construcción del nuevo paradigma integrativo que se plantea, a saber:

- Dos seres-humanos-espirituales generan una inteligencia siempre superior a la de cualquiera de los dos por separado.

- El "otro" es siempre una oportunidad, en una ética integrativa, para la construcción colectiva, y no como en la ética competitiva, un contrincante a quien debemos vencer.

- La inteligencia la comprendemos, en síntesis, como la capacidad de aprovechar información, conocimiento, estructura de pensamiento, praxis o vivencia efectiva y universo emocional para la resolución de conflictos, problemas o desarrollos creativos o lúdicos.

- El retorno a la palabra de vida.

En cualquiera de estas síntesis, se encuentran postulados que han sido profundamente meditados, vivenciados y puestos en la práctica de múltiples comunidades y sociedades durante muchos siglos, corroborados actualmente como generadores de procesos aplicados de solución de problemas complejos y que apuntan a producir transiciones desde las estructuras piramidales de nuestra sociedad hasta una nueva versión holomidal ${ }^{13}$.

Para el caso del retorno a la palabra, el pensamiento integrativo coincide en casi todas sus expresiones como la materia prima que compone los flujos de la comunicación y del compromiso del humano consigo mismo y con el universo. En el maravilloso texto de Carlos Lenkersdorf, Aprender a escuchar: enseñanzas maya-tojolabales (2008), investigadores del pensamiento originario maya viviente en México hacen una profunda crítica a nuestra sociedad: nunca nos interesamos por "escuchar", porque solo pretendemos ser escuchados, principalmente porque el "otro" es una instancia separada de "mí", un riesgo y con quien hay que competir.

Escuchar se convierte en una forma de aparición del "Otro" en la comprensión de lo que entendemos por lenguaje. Procede del órgano de la lengua que es la que produce la palabra, pero el lenguaje está compuesto por dos realidades: palabras que se hablan y que se escuchan. Ambas se complementan mutuamente. Para el occidental el escuchar no tiene importancia. Escuchar no es "importante" para nuestra cultura occidental.

13 Como se expresó antes, el concepto de holomidal es propuesto y explicado profundamente por Jean François Noube en su planteamiento de la revolución invisible, y se refiere a una estructura paralela, equitativa, incluyente, en la cual no se puede comprender la existencia de una jerarquía permanente y se remplaza por la idea de un liderazgo temporal, que depende de un requerimiento concreto que el colectivo posee y de la capacidad o destreza del individuo o subcolectivo que enfrente la situación. 
En realidad, no nos interesa prácticamente nada, escuchar, sentir, saborear o tener piedad. Al escuchar, nos fijamos verdaderamente en lo que el otro y los otros quieren decirnos. Aquí se enfatiza la relación de reciprocidad del nosotros, se perciben palabras y sentimientos desde la perspectiva del otro o los otros. Es una capacidad particular que corresponde a la empatía. Es más, los sabios integrativos saben escuchar no solo a los otros, sino también a "lo otro", es decir, a la naturaleza, al viento, a las plantas, los cultivos, las lluvias, pero en aras de este artículo es mejor no ir tan lejos por ahora.

\section{Universidad, investigación y la responsabilidad socioecológica / pensamiento epistémico}

En este panorama, el enfoque integrativo propone modelos de desarrollo a partir de la acción dialógica, paralela e incluyente de todos los saberes existentes en contextos glocales -incluida la academia-, y no la supremacía o la imposición en relaciones de poder y dominación de alguno de ellos sobre los otros. Por ello, este enfoque se fundamenta en una transición equilibrada, concertada y pacífica de una ética de la competitividad hacia una ética integrativa.

Pero ¿cómo desarrollar efectivamente una propuesta como esta? Las reflexiones generalizadas en los contextos contrahegemónicos se fundamentan, principalmente, en develar o hacer visibles las "arquitecturas invisibles"14; en desconfiar de ellas a partir de modelos como el pensamiento crítico; en revisar, desde los dispositivos de dominación, las relaciones de poder, y en identificar los "sensores de dominación" para poder direccionar una "revolución invisible".

El Informe Mundial sobre las Ciencias Sociales 2010: "Las brechas del conocimiento", elaborado por el Consejo Internacional de las Ciencias Sociales en el 2010 a solicitud de la Unesco, convocó a la auto-organización progresiva de las ciencias sociales en el periodo actual. Esto significa una mayor independencia intelectual y una mayor atención hacia el resto del mundo: dualidad que conducirá a una "auténtica contribución a los debates intelectuales e intercambios académicos con los científicos sociales alrededor del mundo". Esta necesidad coincide con el llamado de las Asociaciones Regionales de las Ciencias Sociales por una mayor autonomía e influencia de la investigación producida en cada región.

En este sentido, la búsqueda de maneras de construir el nuevo paradigma requiere de innovadoras y originales formas de abordaje de las realidades locales y globales, y sobre todo, de comprender la subjetividad y su integralidad sistémica con todo el planeta. Una comprensión de un sujeto más integral -que denominamos ser-humano-espiritual-, que a diferencia de la concepción antropocéntrica del yo occidental, se refiere a un ser integrado, constitutivo y constituyente de su microentorno, que a su vez conforma integralmente y es conformado por la 
conjunción de los diversos micro y macroentornos. La construcción de sentido y el marco de realidad-verdad de un ser-humano-espiritual están constituidos por múltiples interrelaciones (lo que se conoce como alteridad ${ }^{15} \mathrm{u}$ otredad), para cuya comprensión hemos dividido en cuatro tipos de relacionamientos: con los otros $^{16}$, con lo otro ${ }^{17}$, consigo mismo ${ }^{18}$ y con lo trascendente.

El papel de la formación y la educación, ante estas nuevas perspectivas, contiene una transformación sustancial. De una ética de la competitividad individual a una colectiva e integrativa, constituida por valores y principios fundamentados en priorizar el interés colectivo sobre el individual. La solidaridad, la cooperación, la inclusión, la valoración y la legitimación del "otro" diferente, así como la comprensión ecosistémica ampliada de nuestra interdependencia y corresponsabilidad con los ecosistemas y el medio ambiente, etc., invitan a las instituciones educativas, a los centros de investigación y a todas las instancias relacionadas con la formación, a establecer mecanismos de generación de pensamiento propio, integrativo y con tendencia a la consolidación de diálogos de saberes con los otros pensamientos no occidentales: las sabidurías campesinas, las originarias, las milenarias, las de la periferia, las étnicas, las emergentes, las alternativas, etc.

Desde esta perspectiva, la propuesta integrativa científica denominada inteligencia colectiva se presenta como un potente instrumento de generación de pensamientos y sabidurías integrativas propias, a fin de propiciar el diálogo entre saberes interdisciplinares, y entre estos y los a-disciplinares, con lo cual se posiciona como un valioso instrumento de transversalidad sistémica en donde sea implementada. Así mismo, permite construir, en conjunto, una forma de ser sujetos constructores de nuevas realidades, con la capacidad de transformar los diversos ámbitos del ser en todas sus diversas y complejas dimensiones.

La perspectiva disciplinar de la inteligencia colectiva es muy reciente y requiere -para la consolidación como una estrategia transversal a la ECSAH de la UNAD- de un profundo análisis de las tendencias de los desarrollos aplicados y exitosos que esta viene gestando a nivel internacional, pero haciendo énfasis en los procesos latinoamericanos, particularmente en Colombia, e identificando nuevos autores, tendencias, corrientes y experiencias

15 El antropólogo Michael Taussig plantea, en su texto Mimesis y alteridad. Una historia particular de los sentidos (1993), que la alteridad no es un objeto o una cosa, sino que es básicamente una trama de relaciones. Esa trama a la que se refiere Taussing es un sistema de relacionamientos tipificados. texto La conquista de América: el problema del otro (1987) y el trabajo de Gabriel Bello Regera en La construcción ética del otro (1997). En esta tipología entran además todas las fundamentaciones, como las que elabora Aníbal Quijano, y en general, las reflexiones sobre el poscolonialismo, lo subalterno y algunos orientalistas.

17 Para el relacionamiento con "lo otro", nos apoyamos básicamente en dos autores: Edgar Morín con su texto Tierrapatria (1993) y Fritjof Capra con su texto La trama de la vida. Una nueva perspectiva de los sistemas vivos (1998). El concepto de integralidad del planeta como cuerpo viviente o Gaia y la visión sistémica de la vida.

18 Tomamos el concepto de símbolo desde la perspectiva de la psicología analítica de Carl Gustav Jung, pionero de la psicología profunda, que enfatiza la conexión funcional entre estructura de la psique y la de sus productos o manifestaciones culturales. Incluye metodologías y nociones procedentes de la antropología, la alquimia, los sueños, el arte, la mitología, la religión y la filosofía. 
efectivas en proceso que se vienen dando, sobre todo, en Estados Unidos, Francia, Canadá, México y Argentina. El objetivo es que, a partir de allí, se genere una serie de espacios de encuentro con expertos y organizaciones que vengan aplicando este modelo científico, a fin de propiciar diálogos significativos que potencien la producción de pensamiento propio Unadista.

A partir de la necesidad que la UNAD y la ECSAH tienen de dar mayores niveles de profundización y comprensión sociocultural, política y ambiental a los principios rectores expuestos en el Proyecto Académico Pedagógico y Solidario - PAPS 3.0 -solidaridad, democracia, equidad-; y a partir de su necesidad de recuperar los liderazgos y reconocimientos nacionales y globales enmarcados en los cinco focos centrales de acción de la rectoría (inclusión, innovación, investigación, desarrollo regional-léase local o de territorialidades-y proyección comunitaria e internacionalización), se hace necesaria una indagación muy profunda sobre la praxis del trabajo colaborativo, la construcción de conocimiento colectivo, y los mecanismos prácticos y teóricos de lo que significa la interrelación hermenéutica entre "lo solidario", "lo incluyente o democrático" y "lo equitativo o justo", en contextos enmarcados en profundos valores y principios éticos y axiológicos de "competencia" y "supervivencia"

\section{Referencias}

Akyeampong, D. (1998). La educación superior y la investigación: desafíos y oportunidades. París: Unesco. Recuperado el 10 de mayo de 2012, de http:// unesdoc.unesco.org/images/0011/001136/113608so.pdf

Alvarado, V. y Manjarrez, M. (2010). Problemas y retos de la investigación en el siglo XXI (el caso de la RIEMS y la conformación de la antropoiética). Avaliação, 15(2), 107-119.

Barbosa, O. (2004). Evolución de una idea: de la cibernética a la cibercultura. Cuadernos de filosofía Latinoamericana, 25(91), 172-179.

Bauman, Z. (1999). La globalización: consecuencias humanas. México D.F.: Fondo de Cultura Económica.

Bello Reguera, G. (1997). La construcción ética del otro. Oviedo: Ediciones Nobel.

Botero, A. (2004). Nuevos paradigmas científicos y su incidencia en la investigación jurídica. Revista de Derecho, (21), 163-199.

Capra, F. (1998). La trama de la vida. Una nueva perspectiva de los sistemas vivos. Barcelona: Anagrama.

Castoriadis, C. (2007). Democracia y relativismo: Debate con el MAUSS, Madrid, Trotta, p. 90 
Cepeda, J. (2004). Cibercultura y filosofía latinoamericana: una indagación sobre páginas web en lengua castellana. Trabajo monográfico (inédito).

Cerón, E. (2011). Del diálogo de saberes al diálogo de ignorancias. Reflexiones para politizar la acción pedagógica y pedagogizar la acción política. Revista Sustentabilidad(es), (4).

Chomsky, N. (s. f.). El control de nuestras vidas. Recuperado de http://www. inventati.org/ingobernables/textos/anarquistas/chomsky-control.pdf

Cobo Romaní, C. y Pardo Kuklinski, H. (2007). Planeta Web 2.0. Inteligencia colectiva o medios fast food. México D.F.: Flacso.

De Kerckhove, D. (1998). Inteligencia conectada y mente colectiva. Revista de Occidente, (207), 32-42.

- (1999). La piel de la cultura: investigando la nueva realidad electrónica. Barcelona: Gedisa.

Díaz E. (1993). Michel Foucault, los modos de subjetivación. Buenos Aires: Editorial Almagesto.

Escobar, A. (2005). Bienvenidos a cyberia. Notas para una antropología de la cibercultura. Revista de Estudios Sociales, (22), 15-18. Recuperado de: http:// res.uniandes.edu.co/view.php/322/view.php

Estalella, A. y Ardébol, E. (2011). E-research: desafíos y oportunidades para las ciencias sociales. Convergencia. Revista de Ciencias Sociales, (55), 87-111.

Foucault, M. (1968). Las palabras y las cosas. Buenos Aires: Siglo XXI.

. (1981). Subjectivité et vérité. En Annuarie du College de France, 81e année, Historie des systèmes de pensé, année1980-1981 (pp. 385-389). París: s. 1. Recuperado de http://historiasenconstruccion.wikispaces.com/file/view/ MF+-+Subjetividad+y+verdad.pdf

García Canclini, N. (1990). Culturas híbridas, estrategias para entrar y salir de la modernidad. México D.F.: Editorial Grijalbo.

Geertz, C. (2001). La interpretación de las culturas. Barcelona: Editorial Gedisa.

Ghiso, A. (2000). Potenciando la diversidad. Recuperado de http://bibliotecadigital.conevyt.org.mx/colecciones/documentos/potenciando_diversidad.pdf

Gibbons, M. (1998). Pertinencia de la educación superior en el siglo XXI. Recuperado el 10 de mayo de 2012, de http://www.humanas.unal.edu.co/ contextoedu/docs_sesiones/gibbons_victor_manuel.pdf

González, García, M. I., López Cerezo, J. A., y Luján López, J. L. (1996). Ciencia, tecnología y sociedad. Una introducción al estudio social de la ciencia y la tecnología. Madrid: Editorial Tecnos. 
Gordillo, M. (coord.). (2009). Educación, ciencia, tecnología y sociedad. Madrid: OEI. Recuperado el 19 de agosto de 2011, de http://www.oei.es/DOCUMENTO 3caeu.pdf

Herrera, D. (1998). Teoría social de la ciencia y la tecnología. Bogotá: UNAD.

Kuhn, T. (1962). La estructura de las revoluciones científicas. México D.F.: Fondo de Cultura Económica.

Lander, E. (2000). Ciencias sociales: saberes coloniales y eurocéntricos. En Lander, E. (comp.). La colonialidad del saber: eurocentrismo y ciencias sociales. Perspectivas latinoamericanas. Buenos Aires: Clacso. Recuperado de http://biblioteca.clacso.edu.ar/gsdl/collect/clacso/index/assoc/D1334.dir/lander.pdf

Lander, E. (2004). Universidad y producción de conocimiento. En Sánchez Ramos, I. y Sosa Elízaga, R. América Latina: los desafíos del pensamiento crítico. México D.F.: Siglo XXI.

Lenkersdorf, C. (2008). Aprender a escuchar: enseñanzas maya-tojolabales. México D.F.: Plaza y Valdés.

Lévy, P. (1999). ¿Qué es lo virtual? Barcelona: Ediciones Paidós.

Lévy, P. (2004). Inteligencia colectiva: por una antropología del ciberespacio (Felino Martínez Álvarez, trad.). Washington: s. 1. Recuperado de http://inteligenciacolectiva.bvsalud.org/public/documents/pdf/es/inteligenciaColectiva. pdf

Lévy, P. (2007). Cibercultura. La cultura de la sociedad digital. México D.F.: Anthropos.

López Cerezo, J. A. y Luján, J. L. (2000). Ciencia y política del riesgo. Madrid: Editorial Alianza.

López, G. (2002). Sabiduría, poder y comprensión. América se repiensa desde sus orígenes. Bogotá: Siglo del Hombre Editores.

Morín, E. (2007). El hombre y la muerte. Madrid: Editorial Kairós.

Morin, E. y Kern, A. (1993). Tierra-patria. Madrid: Editorial Kairós.

Marin, L. F. (2007). La noción de paradigma. Signo y Pensamiento, 26(50), 34-45. Recuperado de http://www.redalyc.org/pdf/860/86005004.pdf

Méndez Stingl, R. y Álvarez Revilla, A. (1999). Educando en valores a través de "Ciencia, Tecnología y Sociedad". Bilbao: Desclée de Brouwer.

Nicolescu, B., et al. (1994). Manifiesto de la transdisciplinariedad. Portugal: 6 de diciembre de 1994. (Víctor Morales, trad.). Caracas: Universidad de Venezuela. 
Noubel J. F. (2004). Inteligencia colectiva, la revolución invisible. Recuperado de: https://solocreatividad.files.wordpress.com/2011/01/intelco.pdf

Núñez, J., (2001). Ciencia y cultura en el cambio de siglo. A propósito de C.P Show. En López Cerezo, J. A. y Sánchez, J. M. Ciencia, tecnología, sociedad y cultura en el cambio de siglo. Madrid: Biblioteca Nueva.

Observatorio Indígena de Políticas Públicas de Desarrollo y Derechos Étnicos. (2007). Etnias y Política. Revista del Observatorio Indígena de Políticas Públicas de Desarrollo y Derechos Étnicos, (4). Bogotá, abril de 2007. Recuperado de http://www.observatorioetnicocecoin.org.co/descarga/etnias4.pdf

Organización de Estados Iberoamericanos (OEI). (2001). Ciencia, tecnología y sociedad. Recuperado el 02 de agosto de 2010, de: http://www.oei.es/cts.htm

Organización Nacional Indígena de Colombia (ONIC). (2011). Revista ONIC. Bogotá: Autor.

Pareja, E. y Sánchez, J. (1998) Una aproximación a los estudios de Ciencia, Tecnología y Sociedad (CTS). Recuperado el 16 de agosto de 2011, de http://www.ugr. es/ eianez/Biotecnologia/cts.htm

Pérez, C. y Echeverry, J. (2010). Memorias Primer Encuentro Amazónico de Experiencias de Diálogo de Saberes. Bogotá: Universidad Nacional de Colombia.

Rheingold, H. (2004). Multitudes inteligentes. La próxima revolución social (Smart Mobs) (Marta Pino Moreno, trad.). Barcelona: Gedisa.

Ruíz, E., (2004). La desconocida y manipulada relación entre ciencia social e ideología. En Sánchez Ramos, I. y Sosa Elízaga, R. América Latina: los desafíos del pensamiento crítico. México: Siglo XXI.

Savater, F. (1999). Las preguntas de la vida. Barcelona: Editorial Ariel.

Schlierf, K., (2007) La enseñanza Ciencia, Tecnología y Sociedad (CTS) en el entorno universitario politécnico. La metodología de la descripción de controversias en la Escuela de Minas de París. Recuperado el 13 de junio de 2011, de http://www.revistacts.net/files/Volumen $\% 205 \% 20-\% 20$ N\%C3\%BAmero\%2015/schlierf_edit.pdf

Sierra, L. I. (2009). Reseña de "Multitudes inteligentes. La próxima revolución social (Smart Mobs)", de Howard Rheingold. Signo y Pensamiento, 28(54), 382-383. Recuperado de http://www.redalyc.org/pdf/860/86011409027.pdf

Taussig, M. (1993). Mímesis y alteridad. Una historia particular de los sentidos. Londres: Routledge.

Todorov, T. (1987). La conquista de América: el problema del otro. México D.F.: Siglo XXI. 
Tovar, L. (2001). Notas sobre la transformación de los saberes sociales en Colombia. Expedición científica y cultural. Revista Virtual de la UNAD, (3). Recuperado de http://www.bdigital.unal.edu.co/16188/1/11061-26360-1PB.pdf . (2004). Seminario Interdisciplinario de Docentes: investigación y saberes sociales. Bogotá: UNAD.

Unesco. (2010). Informe Mundial sobre las Ciencias Sociales 2010: “Las brechas del conocimiento". París: Autor. Recuperado de http://unesdoc.unesco.org/ images/0021/002173/217366s.pdf

Wilches, L. E. (2009). Al reencuentro con "lo otro" alteridad y sistema de pensamiento de la cultura ika o arahuaco en el Caribe colombiano (Tesis de maestría, Universidad Nacional de Colombia, Bogotá).

Williams, R. (1994). Sociología de la cultura. Barcelona: Ediciones Paidós. . (2001). La larga revolución. Buenos Aires: Editorial Nueva Visión.

Zemelman, H. (1998). Sujeto: existencia y potencia. México D.F.: Anthropos. . (2003). Los horizontes de la razón I. Madrid: Anthropos.

- (2011). Historia y uso crítico del lenguaje. Revista Latinoamericana de Metodología de las Ciencias Sociales, 1(1). Recuperado de http://www.relmecs. fahce.unlp.edu.ar/article/view/v01n01a03/106 